\title{
Improving Hybrid Models For Precipitation Forecasting By Combining Nonlinear Machine Learning Methods
}

\section{Laleh Parviz}

Azerbaijan Shahid Madani University

Kabir Rasouli

University of British Columbia

Ali Torabi ( $\nabla$ ali.torabihaghighi@oulu.fi )

University of Oulu https://orcid.org/0000-0002-5157-0156

\section{Research Article}

Keywords: Improved hybrid model, Precipitation, Forecast combination, Support vector regression

Posted Date: August 17th, 2021

DOI: https://doi.org/10.21203/rs.3.rs-779973/v1

License: (c) (i) This work is licensed under a Creative Commons Attribution 4.0 International License.

Read Full License 


\title{
Improving hybrid models for precipitation forecasting by combining nonlinear machine
} learning methods

\author{
Laleh Parviz ${ }^{1}$, Kabir Rasouli ${ }^{2}$ and Ali Torabi Haghighi ${ }^{3, *}$ \\ ${ }^{1}$ Faculty of Agriculture, Azerbaijan Shahid Madani University, Tabriz, East Azerbaijan, Iran \\ ${ }^{2}$ Department of Forest Resources Management, University of British Columbia, Vancouver, BC, \\ Canada \\ ${ }^{3}$ Water, Energy and Environmental Engineering, University of Oulu, Oulu, Finland \\ *Corresponding author: Ali Torabi Haghighi email: ali.torabihaghighi@oulu.fi
}

\begin{abstract}
Precipitation forecast, especially on monthly and annual scales, is a key for optimal water resources management and planning, especially in semiarid climates with scarce water. The traditional hybrid models, in which two statistical models are used to separate and simulate linear and nonlinear components of precipitation time series, are still unable to provide accurate precipitation forecasts. This research aims to improve hybrid forecast models by combining one linear model and three nonlinear models with two preprocessing configurations: 1) using residuals of a linear model, representing the nonlinear component with different time steps and 2) using original time series of observations with different time steps, linear model simulations and residuals. Gene Expression Programming (GEP), Support Vector Regression (SVR) and Group Method of Data Handling (GMDH) models were used individually as in the traditional hybrid models and combinedly as in the proposed hybrid models in this study. The performance of the hybrid models was improved by different methods such as inverse variance (Iv) as an error-based method, least square regression, genetic algorithm and SVR. Two weather stations of Tabriz (annual) and Rasht (monthly) in Iran were selected to test the developed models. The results showed that Theil's coefficient, UII, decreased in configuration one for the Tabriz station by $9 \%$ and $15 \%$ for SVR and GMDH relative to GEP, suggesting that these two models performed better than GEP in the precipitation forecast. The error criteria used in developing the proposed hybrid models with all forecast combination methods better represent observations than the hybrid model. MSE decreased by $67 \%$ and Nash Sutcliffe increased by $5 \%$ in the Rasht station in configuration two when we combined the three models using GA to obtain the improved hybrid model relative to the hybrid model combined with SVR. Generally, the hybrid models when SVR, the error based methods and GA were incorporated showed better performance than traditional hybrid models. The developed models have implications for modeling highly nonlinear systems using full advantages of machine learning methods.
\end{abstract}

Keywords: Improved hybrid model, Precipitation, Forecast combination, Support vector regression 


\section{Introduction} Accurate precipitation forecast remains challenging despite enormous attempts to improve weather and climate models (Adhikari and Agrawal 2014), which is partly due to its complex nature. Stochastic models were extensively used for precipitation forecasting. The models such as autoregressive integrated moving average (ARIMA), seasonal ARIMA (SARIMA), vector autoregression (VAR) and generalized autoregressive conditional heteroskedasticity (GARCH) are among the common stochastic models (Wang et al., 2013; Papalaskaris et al., 2016; Murthy et al., 2018; Bouznad et al., 2020; Nugroho et al., 2014; Mehdizadeh et al., 2017). Despite the popularity of these models, their applications in representing a nonlinear characteristic of precipitation were not successful over the past. It is expected that the linear models not to be able to capture complex phenomena (Chen and Wang 2007). Alternatively, some machine learning techniques such as support vector regression (SVR) were used to solve nonlinear problems such as precipitation forecasting (Hamidi et al., 2015; Shenify et al., 2016), streamflow forecasting (Rasouli et el., 2012; 2020), freshwater-saltwater interface fluctuations prediction (Yoon et al., 2017) and groundwater level estimation (Moravej et al., 2020). The machine learning techniques also require a rigorous search algorithm to obtain optimal hyperparameters (Zeynoddin et al.,2018). According to the strengths of both linear and nonlinear models, it is reasonable to use a combination of models for forecasting problems. In this regard, hybrid models were introduced in the literature to assess the forecast accuracy compared to individual models (Chen and Wang 2007). A hybrid model, combining linear and nonlinear components of a complex process such as precipitation, can perform better than each of the composing models. In hybrid systems, nonlinear components can be obtained from the difference of observed and linear components, so called error series or residual time series (Chen et al., 2020). A combination of SARIMA and support vector machines (SVM) decreased mean absolute percentage error (MAPE) by $43 \%$ relative to the case when only SARIMA was used and by $32 \%$ relative to the case when only SVM was used in forecasting the production values of machinery industry (Chen and Wang, 2007). Another example of hybrid models was ARIMA and artificial neural network (ANN) models for particulate matter forecasting in urban areas (Diaz-Robles et al., 2008). A combination of SARIMA and ANN models was used to forecast monthly inflow, and the combined model had a high coefficient of determination relative to these two composing models (Moeeni et al., 2017). Another hybrid model was developed based on ARIMA coupled with ANN using GA to forecast the production value of 
the mechanical industry (Liang 2009). GA was used to optimize the ANN parameters, such as number of hidden neurons. The improvement of traditional hybrid model, especially in nonlinear part of time series, can significantly affect the accuracy of forecasts. Some decomposed time series from observed time series are often highly nonlinear and the use of a single nonlinear model for accurate forecasting of those time series is challenging. Also, forecasting residuals with large errors make the entire forecast series problematic (Mo et al., 2018). Therefore, there is a need to combine the forecast of nonlinear time series and avoid the large errors in some of the subseries by giving less weights to them. For example, the group method of data handling (GMDH) neural network was used to combine the nonlinear models (e.g., SVR, back-propagation (BP) neural network and genetic programming (GP)). The performance of the more recently improved hybrid models was better than individual models such as SARIMA or previously used hybrid models such as SARIMA-SVR and SARIMA-BP (Mo et al., 2018). Another approach to couple nonlinear time series is to use a weighted combination of the forecasts. In this method, the combination is based on assigning proper weights to the single models to better extract the right information from the time series with the right individual models (Song and Fu 2020). One of the important challenges related to forecasting combination is the precise selection of weights (Wang et al., 2019), which affects the accuracy of the forecasts. The simple average (Timmermann 2006), the error-based methods (Adhikari and Agrawal 2014, Song and Fu 2020), the least square regression method (Frietas and Rodrigues 2006), and the differential weighting methods (Winkler and Makridakis 1983a; Chen et al., 2004) are a few examples of weighted combination methods. The main purpose of this research is to improve a hybrid model in the field of precipitation forecasting with emphasis on the nonlinear part of time series (in two time steps: annual and monthly). ARIMA and SARIMA were used for modeling the linear part of the time series. Individual models such as SVR, GMDH and GEP were applied to model nonlinear patterns of time series. To better capture the complexity of nonlinear patterns of precipitation, instead of one single model, the results of three single models were combined. The error-based methods, the least square regression method, optimization with GA, artificial intelligence techniques such as SVR and ANN were used to combine the three individual models.

(9)

8




\section{2.1. Case Study}

101 To evaluate the performance of the proposed improved hybrid model, precipitation data were used 102 in the Rasht and Tabriz weather stations in the Gilan and East Azerbaijan provinces in Iran with 103 humid and semiarid climates. The mean annual precipitation is $279 \mathrm{~mm}$ in the Tabriz station and $1041278 \mathrm{~mm}$ in the Rasht station. The coefficient of variation for precipitation is 0.25 and 0.2 in Tabriz 105 and Rasht, respectively. Based on the climograph of these stations, monthly precipitation is more 106 variable in the Rasht station than Tabriz station (Figures $1 \mathrm{~b}$ and d). The climate of Rasht and Tabriz 107 regions is very humid and semi-arid based on De Martonne climate classification (De Martonne, 108 1925), wet and cold dry based on Emberger climate classification (Emberger, 1952). 

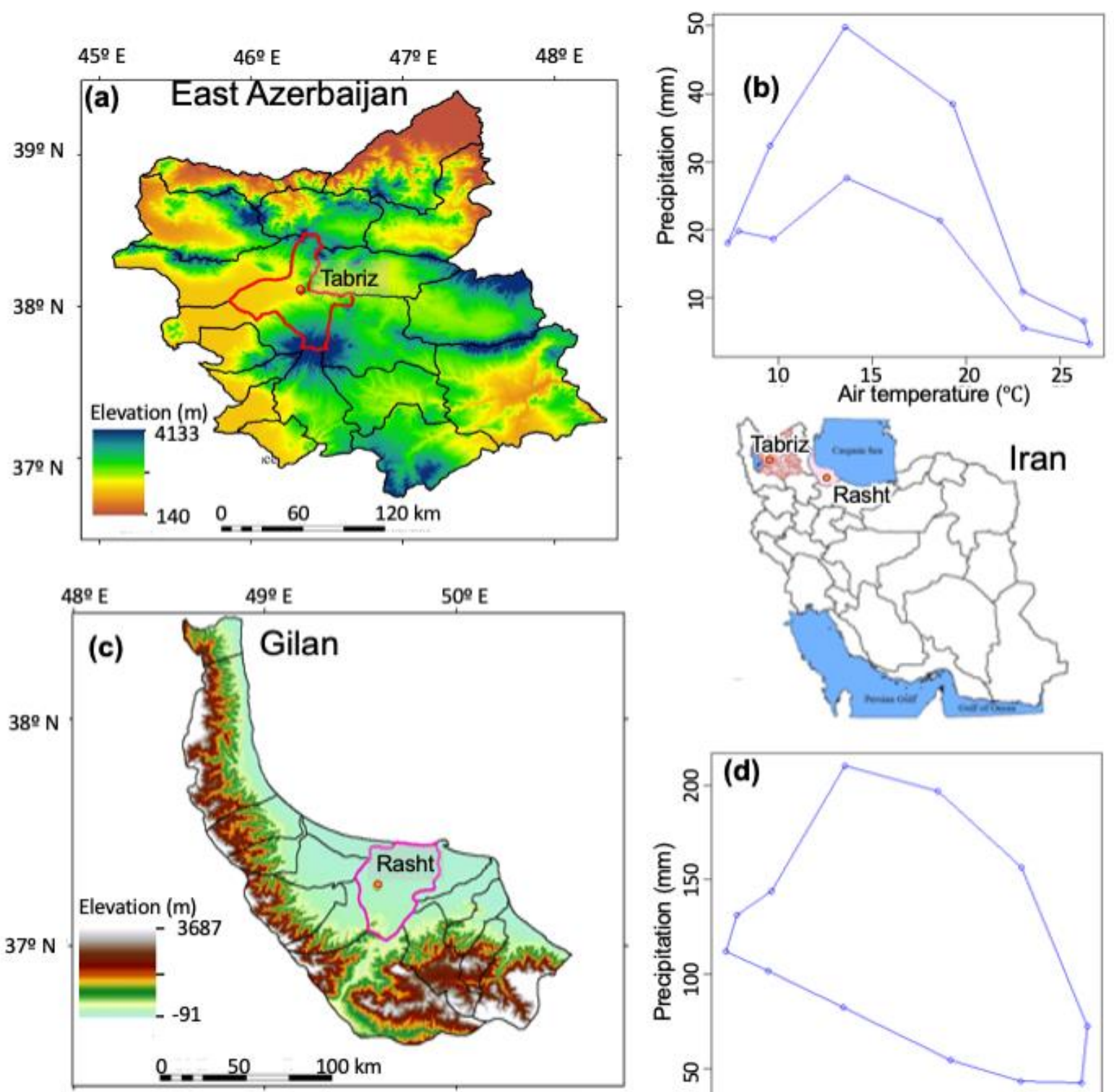

Figure 1: (a) Position and (b) climograph of Tabriz weather station in East Azerbaijan province and (c) position and (d) climograph of Rasht weather station in Gilan province in Iran over the 112 1992-2019 period.

\subsection{Traditional hybrid models based on two linear and nonlinear models}

115 One of the hypotheses related to the time series structure is the separation of time series into two

116 linear and nonlinear components, as in Eq. 1 (Chen and Wang, 2007). The performance of the

117 hybrid model depends on the estimated residuals, which are obtained from the difference between 118 observations and linear components estimated by the linear model (Eq. 2). The nonlinear 
119

120

121

122

123

124

125

126

127

128

129

130

131

132

133

134

135

136

137

138

139

140

141

142

143

144

145

146

147 148

component, therefore, is estimated with different models and configurations. Finally, the forecasted time series are obtained by summing estimated linear and nonlinear components (Zhang 2003).

$y_{t}=L_{t}+N_{t}$

$e_{t}=y_{t}-\hat{L}_{t}$

where $L_{t}$ is the linear component, $N_{t}$ denotes the nonlinear component, $\varepsilon_{t}$ is the error term or the residual component. The linear part of the time series can be modeled with ARIMA or SARIMA, while the modeling of the nonlinear component can be done with machine learning techniques such as SVR or ANN (Figure 2).

\subsubsection{Modeling the linear component of time series}

The forecasting processes of stochastic models such as ARMA, ARIMA, and SARIMA are based on the Box-Jenkins methodology (1976). The SARIMA model is an extension of the ARIMA model to represent seasonal variations. ARIMA and SARIMA models can be expressed as in Eqs. 3 and 4.

$a_{p}(B)(1-B)^{d} Y_{t}=\theta_{0}+e_{q}(B) r_{t}$

$a_{p}(B) A_{p}\left(B^{s}\right)(1-B)^{d}\left(1-B^{s}\right)^{D} Y_{t}=\theta_{0}+e_{q}(B) E_{Q}\left(B^{s}\right) r_{t}$

where $a_{p}(B), e_{q}(B)$ and $A_{p}\left(B^{s}\right)$ and $E_{Q}\left(B^{s}\right)$ are polynomial with orders $p, q$ and $P, Q$ for autoregressive and moving average terms, non-seasonal and seasonal terms, respectively. (1-B) and $\left(1-B^{s}\right)$ are the regular and seasonal differencing operates, respectively. $d$ is the number of differencing operations, $D$ is the number of seasonal differencing. $Y_{t}$ is the observed value at time $\mathrm{t}, \theta_{0}$ is a fixed term, and $r_{t}$ is a random error (Nwokike et al., 2020).

\subsubsection{Individual models for modeling nonlinear components of time series}

\section{Support Vector Machine}

SVM is a machine learning methodology (Vapnik, 1995), which is widely used in classification problems, regression estimation, pattern recognition and probability density function estimation. The general form of SVM for regressions is expressed as: 
151 where $\mathrm{f}(\mathrm{x})$ is the fitted function, $\omega$ is the weight of the input vector, $\varphi(x)$ is the map or kernel 152 function to transform $x_{i}$ to a high-dimensional feature space, and $b$ is an adjustable factor. The 153 penalty parameter in SVM can control the tolerance of the systematic outliers (Liu et al., 2014). 154 One step in training a SVR model is to select the best kernel functions. Usually, radial basis and 155 sigmoid functions show better performance than linear kernels (Xu et al., 2019; Zhu et al., 2018).

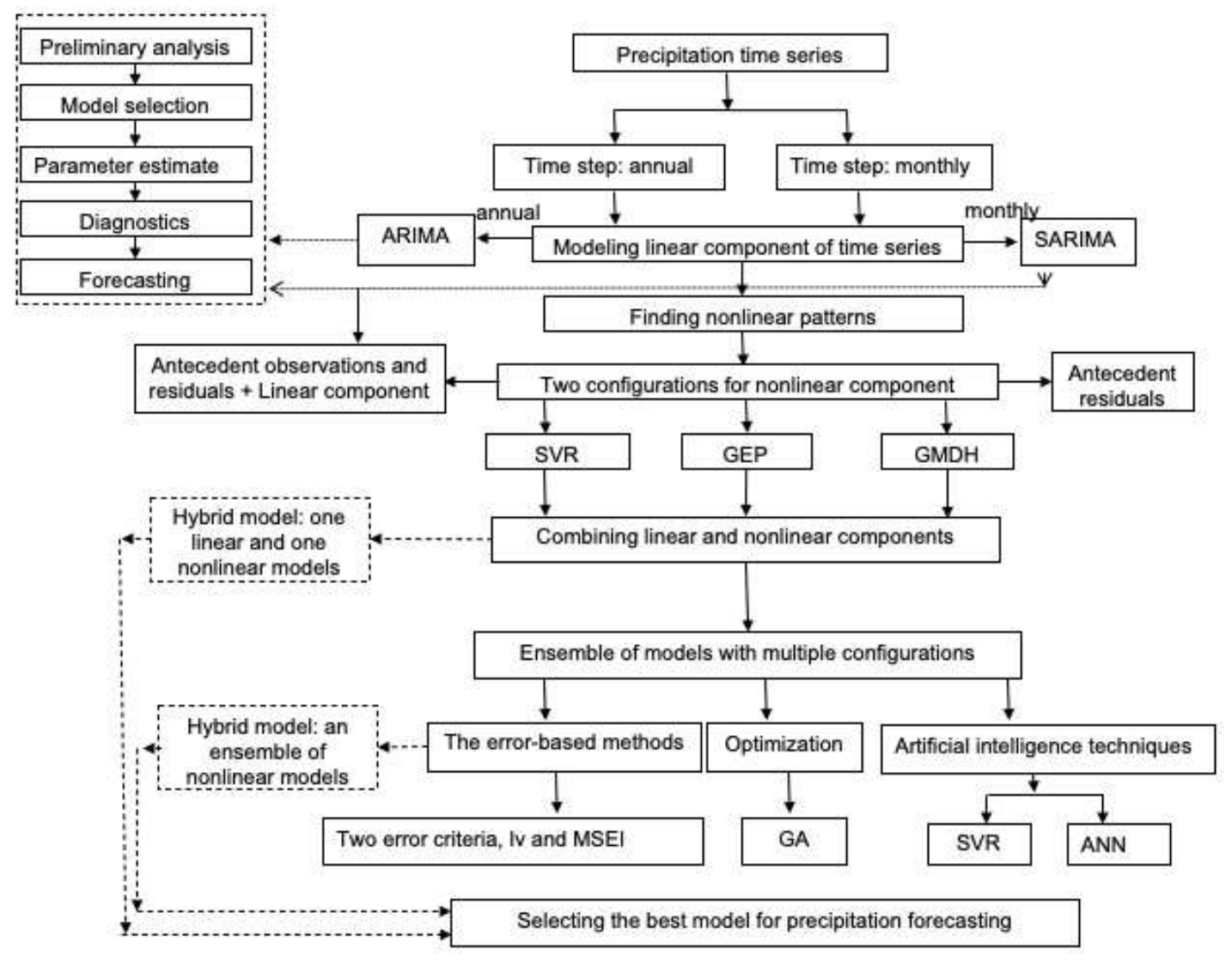

Figure 2: The structure of hybrid model with linear and nonlinear components and improved hybrid model with combined weighted forecasts instead of using one single model in nonlinear component.

$162 \mathrm{GMDH}$, or polynomial neural network, is a self-organization technique, which is used for simulating complex systems (Jeddi and Sharifian, 2020; Mo et al., 2018). The model has a set of 
neurons in which different pairs in each layer are connected through a polynomial and in each layer, new neurons are built up for the next layer. The complicated discrete form of the Volterra functional series (Volterra, 1959), such as in Eq. 6 were applied for general connection between the input and output variables which is also known as the Kolmogorov-Gabor (K-G) polynomial (Mo et al., 2018).

For modeling nonlinear input/output relationships, the Volterra series can be used (Marzocca et al., 2008).

$y=a_{0}+\sum_{i=1}^{n} a_{i} x_{i}+\sum_{i=1}^{n} \sum_{j=1}^{n} a_{i j} x_{i} x_{j}+\sum_{i=1}^{n} \sum_{j=1}^{n} \sum_{k=1}^{n} a_{i j k} x_{i} x_{j} x_{k}+\ldots$

where $y$ is output variables, $x$ is input variables, $a$ is a coefficient.

\section{Gene Expression Programming}

GEP, which was introduced as a new function model mining algorithm by Ferreira (2001), was based on Darwin's theory of evolution. GEP superiority to GA and GP is its convergence speed and capability in solving complex problems. Chromosomes and expression tress are the main components of GEP. The start of GEP is based on the random generation of chromosomes that form one or more genes in the name of sub-expression tree (sub-ET). The best solution is to link the sub-ETs through algebraic or Boolean (AND, OR, NOT) functions. Each sub-ET is a part of the solution with less complexity and provides information about the given process less than the corresponding expression tree. Finally, the power of GP blocks is applied with GEP to model nonlinear pattern of a complex system through a multi gene structure (Deng et al., 2018- Yousefi et al., 2017; DanandehMehr, 2018).

\subsection{Improved hybrid models based on one linear model and a combination of nonlinear models}

One approach to increase the efficiency of the traditional hybrid models in simulating the nonlinear component is to utilize a combination of individual models to forecast residuals instead of using a single model (Mo et al., 2018). In this study, GEP, SVR and GMDH were used to model the residuals with two configurations, composed of residual subseries with different antecedent time steps $(t-1),(t-2),(t-3)$ or composed of linear component $\left(\hat{L}_{t}\right)$, original observed precipitation time series with antecedent time steps $\left(y_{t-1}\right.$ or $\left.y_{t-12}\right)$ and residual subseries with different time steps, and 
194 the forecasts of mentioned models were combined by different strategies such as the least square 195 regression method to obtain a weighted combination of the three individual models. The steps of 196 the improved hybrid model structure proposed in this study include (Figure 2):

197

198 1) Stochastic models (ARIMA for annual and SARIMA for monthly time series) are applied to 199 model linear patterns of the precipitation time series $\left(\hat{L}_{t}\right)$.

200 2) Calculating error or residual subseries $\left(e_{t}\right)$ based on Eq. 2 using observed values $\left(y_{t}\right)$ and 201 estimated linear time series in step $1\left(\hat{L}_{t}\right)$.

202 3) Determining two configurations to model residual subseries for annual $\left(y_{a n}\right)$ and monthly $\left(y_{m o n}\right)$ 203 precipitation. In these configurations, $\varepsilon_{t}$ denotes the random error.

- Configuration one based on residual subseries with different antecedent time steps $(t-1),(t-2)$, $(t-3)$ :

$e_{t}=f\left(e_{t-1}, e_{t-2}, e_{t-3}\right)+\varepsilon_{t}$

208 209

210

211

213

214

215 216

217

218

219

220

221
- Configuration two based on linear component $\left(\hat{L}_{t}\right)$, original observed time series with antecedent time steps $\left(y_{t-1}\right.$ or $\left.y_{t-12}\right)$ and residual subseries with different antecedent time intervals from step 1:

$$
\begin{aligned}
& y_{t-a n}=f\left(y_{t-1}, y_{t-2}, \hat{L}_{t}, e_{t-1}, e_{t}\right)+\varepsilon_{t} \\
& y_{t-m o n}=f\left(y_{t-1}, y_{t-12}, \hat{L}_{t}, e_{t-1}, e_{t}\right)+\varepsilon_{t}
\end{aligned}
$$

4) Forecasting residual subseries with two configurations in step 3 from each of GEP, SVR and GMDH nonlinear models. In the configurations with residual subseries, $e_{t}$, as the output, it is necessary to sum up the estimated linear and nonlinear components.

5) Obtaining weighted forecasts $\widehat{y}_{t}^{n, c}$ : We combine the forecasts of the three nonlinear models with the most reasonable weights (Song and Fu 2020). The general structure of weighted forecasts is expressed in Eq. 7:

$$
\hat{y}_{t}^{n, c}=\sum_{i=1}^{m} w_{i} \cdot \hat{y}_{t}^{n, i, c}
$$


$222 n=\left(\begin{array}{cc}1 & \text { Monthly } \\ 2 & \text { Annual }\end{array}\right), i=\left(\begin{array}{cc}1 & G E P \\ 2 & S V R \\ 3 & G M D H\end{array}\right), \quad c=\left(\begin{array}{l}\text { Configuration 1 } \\ \text { Configuration 2 }\end{array}\right)$

223

224 where $w_{i}$ is the weight coefficient of the $i$ th individual model and $m$ is the number of models $225 \quad\left(\sum_{i=1}^{m} w_{i}=1\right)$.

226

227 The inverse variance method, Iv, as in Eq. 8, is one of the weighted combining methods that are 228 based on the inversion of the forecast errors of the corresponding models. Another method is the 229 mean square error inverse, MSEI, as in Eq. 9, which can be categorized as an error-based method 230 (Adhikari and Agrawal, 2014; Song and Fu, 2020). The forecast errors (Eqs. 8 and 9) are calculated 231 by obtaining the sum of squared error (SSE) in Eq. 8 and symmetric mean absolute percentage 232 error (SMAPE) in Eq. 9, which are defined as in Eqs. 10-11.

233

$234 \quad w_{i}=\frac{e_{i}^{-1}}{\sum_{i=1}^{m} e_{i}^{-1}}$

$235 w_{i}=\frac{e_{i}^{-\frac{1}{2}}}{\sum_{i=1}^{m} e_{i}^{-\frac{1}{2}}}$

$e_{i}=S S E=\sum_{t=1}^{n}\left(y_{t}-\hat{y}_{t}^{n, i, c}\right)^{2}, \quad i=1,2, \ldots, m$

$$
e_{i}=S M A P E=\frac{1}{N} \sum_{t=1}^{n} \frac{\left|y_{t}-\hat{y}_{t}^{n, i, c}\right|}{\frac{y_{t}+\hat{y}_{t}^{n . i, c}}{2}}, \quad i=1,2, \ldots, m
$$

239 The least-square regression (LSR) method was used in this study to minimize the sum of squared 240 error for a linear combination of weighted forecasts. The matrix form of Eq. 7 can be written as in 241 Eq. 12. Therefore, sum of squared error between observed and simulated $\left(y_{i}, \hat{y}_{i}\right)$ values can be 242 described with Eq. 13. At the end, the weights vector can be obtained by minimizing SSE as in Eq. 24314 (Frietas and Rodrigues, 2006; Adhikari and Agrawal, 2014). 
$244 \quad \hat{\boldsymbol{Y}}=\boldsymbol{U} \boldsymbol{W}=\left[\begin{array}{llll}\hat{y}_{1}^{1} & \hat{y}_{1}^{2} & \ldots & \hat{y}_{1}^{m} \\ \hat{y}_{2}^{1} & \hat{y}_{2}^{2} & \ldots & \hat{y}_{2}^{m} \\ \cdot & & & \\ \cdot & & & \\ \cdot & & & \\ \hat{y}_{n}^{1} & \hat{y}_{n}^{2} & \ldots & \hat{y}_{n}^{m}\end{array}\right], w=\left[w_{1}, w_{2}, \ldots, w_{m}\right]^{T}$

$245 \quad S S E=Y^{T} Y-2 W^{T} U^{T} Y+W^{T} U^{T} U W$

$246 W=\left(U^{T} U\right)^{-1} U^{T} Y$

247

248

The GA optimization method was used to find the weights so that the error between observed and

249 simulated precipitation was minimized. The objective function and constraints were expressed as

250 below (Prudêncio and Ludermir, 2006).

251

Minimize :

252

$\operatorname{error}\left(\hat{y}_{t}^{n, c}\right)=\sum_{t=1}^{n}\left(y_{t}-\hat{y}_{t}\right)=\sum_{t=1}^{n}\left(y_{t}-\sum_{i=1}^{m}\left(w_{i} \cdot \hat{y}_{t}^{n, i, c}\right)\right)$

Subject to :

253

$\sum_{i=1}^{m} w_{i}=1$ and $w_{i} \geq 0$

254

255 Additionally, precipitation values forecasted by GEP, SVR and GMDH were used as inputs to the

256 SVR model to improve the forecasts (Eq. 17) further. A sensitivity analysis was carried out based

257 on the type of kernel function and penalty parameter.

258

$259 \quad \hat{y}_{t}^{n, c}=f_{\text {(kernal-penalty) }}\left(\hat{y}_{t}^{n, 1, c}, \hat{y}_{t}^{n, 2, c}, \ldots, \hat{y}_{t}^{n, m, c}\right)$

260

261 An ANN model was also used to improve the forecasts by GEP, SVR and GMDH models (Eq.

262 18). A sensitivity analysis was carried out to obtain optimal number of neurons in the hidden layer 263 and type of activation function.

$264 \hat{y}_{t}^{n, c}=f_{\text {ANN }}\left(\hat{y}_{t}^{n, 1, c}, \hat{y}_{t}^{n, 2, c}, \ldots, \hat{y}_{t}^{n, m, c}\right)$ 


\section{2.4. Performance evaluation of the improved hybrid models}

267 In order to assess the performance of the model, evaluation metrics can be used which some of 268 them are used in this study to compare the performance of hybrid and improved hybrid models.

269 The used criteria or metrics are mean square error (RMSE), relative root mean square error 270 (RRMSE), mean absolute error (MAE), mean square error (MSE), Theil's coefficient of UI, UII, 271 residual predictive deviation (RPD), APB, modified index of agreement $\left(\mathrm{d}_{\mathrm{m}}\right)$, adapted mean 272 absolute percentages error (AMAPE), accuracy improved (AI) and geometric mean error ratio 273 (GMER).

274

$275 \quad R M S E=\frac{1}{N} \sqrt{\sum_{i=1}^{N}\left(O_{i}-P_{i}\right)^{2}}$

276

$R R M S E=\frac{R M S E}{\bar{O}}$

$277 \quad M S E=\frac{\sum_{i=1}^{N}\left(P_{i}-O_{i}\right)^{2}}{N}$

278

$\boldsymbol{U I}=\frac{\left[\sum_{i=1}^{N}\left(P_{i}-O_{i}\right)^{2}\right]^{0.5}}{\left[\sum_{i=1}^{N}\left(O_{i}\right)^{2}\right]^{0.5}+\left[\sum_{i=1}^{N}\left(P_{i}\right)^{2}\right]^{0.5}}$

$279 \quad U I I=\frac{\left[\sum_{i=1}^{N}\left(P_{i}-O_{i}\right)^{2}\right]^{0.5}}{\left[\sum_{i=1}^{N}\left(O_{i}\right)^{2}\right]^{0.5}}$

280

$M A E=\frac{1}{N} \sum_{i=1}^{N}\left|\frac{P_{i}-O_{i}}{O_{i}}\right|$

$281 \quad R P D=\frac{S D}{R M S E}$

282

$N S E=1-\frac{\sum_{i=1}^{n}\left(P_{i}-O_{i}\right)^{2}}{\sum_{i=1}^{n}\left(O_{i}-\bar{O}\right)^{2}}$

283

$A M A P E=\frac{1}{n} \sum_{i=1}^{n}\left(\frac{\left|P_{i}-O_{i}\right|}{\frac{1}{n} \sum_{i=1}^{n} Q_{i}}\right) \times 100$ 
$A P B=\frac{\sum_{i=1}^{n}\left|P_{i}-O_{i}\right|}{\sum_{i=1}^{n} O_{i}} \times 100$

285

$d_{m}=1-\frac{\sum_{i=1}^{n}\left|P_{i}-O_{i}\right|}{\sum_{i=1}^{n}\left(\left|P_{i}-\bar{O}\right|+\left|O_{i}-\bar{O}\right|\right)}$

$286 \quad A I=\frac{S-S_{h}}{S} \times 100$

$G M E R=\exp \left(\frac{1}{n} \sum_{i=1}^{n} \ln \left(\frac{p_{i}}{O_{i}}\right)\right)$

288

289

290

291

292

293

294

295

296

297

298

299

300

301

302

303

304

305

306

307

308

309

where $P_{i}$ is the forecasted value, $O_{i}$ is the observed value, $\bar{O}$ is the mean of observed value, $S$ is $M A E$ of the hybrid model, $S_{h}$ is $M A E$ of the improved hybrid model. Minimum values of $R M S E$, RRMSE, MAE, MSE, $A P B$ and AMAPE indicate the similarity between forecasted and observed values (Ruiz-Aguilar et al., 2014; del Carmen Bas et al., 2017; DanandehMehr, 2018). Theil's coefficient (Theil, 1966; 1961) were used for evaluating models forecasting accuracy (UI) and quality of forecasting ( $U I I) . U I=0$ and $U I I=0$ are indicative of perfect forecasts (Zeynoddin et al., 2018). AI greater than 0 indicates the superior performance of the hybrid model, whereas $A I$ less or equal to 0 shows that the hybrid model does not outperform any individual model (Chen and Zhu 2013). The high NSE indicates the good performance of a model (DanandehMehr 2018) and its value is one for a perfect model. The $R P D$ values are less than 1.4 and greater than two show poor and high performance, respectively. The range of $d_{m}$ is between 0 and 1 , where 1 is an indicative of high performance of the model (Ruiz-Aguilar et al., 2014; Duveiller et al., 2016). GMER > 1 indicates overestimation and GMER $<1$ shows underestimation (Abdelbaki 2016).

\section{Results}

The observation period for precipitation data was 1964-2019 for annual and 2000-2019 for monthly time series. The investigated dataset was split into three subsets, $80 \%$ for calibration, $10 \%$ for validation, and $10 \%$ for verification. In order to check the sufficiency of data length for modeling, the Hurst coefficient (Hurst et al., 1965) was determined 0.8 and 0.6 in Tabriz and Rasht, respectively (when the Hurst coefficient is more than 0.5 data length is sufficient for modeling). 


\subsection{Modeling linear component of precipitation time series}

ARIMA and SARIMA models were applied as linear models for annual and monthly time series, respectively. The presence of seasonality was checked and assured for SARIMA model application in precipitation time series for Rasht station using autocorrelation function and partial autocorrelation function. The autocorrelation function had intervals of 12 , clearly proving that there is a seasonal cycle in the Rasht precipitation. Thus, seasonal differencing in SARIMA model is required. The results of preprocessing steps in precipitation time series analysis were shown in Table 1. The normality tests of Kolmogorov-Smrinov (Kolmogorov,1933) and Shapiro-Wilk tests (Shapiro and Wilk, 1965) confirmed the normality of the Tabriz precipitation time series. The significance level of the normality tests was less than the critical value for the Rasht precipitation time series. Therefore, a transformation of the time series was necessary. After transformation, the significance level of Kolmogorov-Smrinov and Shapiro-Wilk tests improved to reach 0.2 and 0.167, respectively. Furthermore, the Augmented Dickey-Fuller (ADF) test (Said and Dickey 1984) was used to check the stationarity of the time series. The statistics $t_{\alpha}$ was greater than the critical values (-3.6 and -2.57 for Tabriz and Rasht at $1 \%$ level), suggesting that both time series were non-stationary (Table 1). The Mann-Kendall test (seasonal Mann-Kendall test for Rasht time series) showed a significant decreasing trend in precipitation observed in both stations. The ADF test was checked once again after applying for differencing order on time series and the statistics $t_{\alpha}$ was recalculated and found to be less than the critical values and hence, the precipitation values to become stationarity. To find the orders of models, some range of parameters order is examined, ARIMA and SARIMA: $a \in\{0,1,2,3,4,5,6\}, e \in\{0,1,2,3,4,5\}$ and $a, A, e, E \in\{0,1,2,3\}$.

Out of many optional models, $\operatorname{SARIMA}(0,1,1) \times(0,1,1) 12$ and $\operatorname{ARIMA}(0,1,2)$ had the minimum values of SBC and BIC for Rasht and Tabriz precipitation (Table1). The SBC decreased from $\operatorname{ARIMA}(4,1,1)$ to $\operatorname{ARIMA}(0,1,2)$ by $6.6 \%$ and from $\operatorname{SARIMA}(1,1,0)(0,1,0)_{12}$ to SARIMA $(0,1,2)(0,1,1)_{12}$ by $28.5 \%$. The significance levels of the Shapiro-Wilk test for Rasht and Tabriz residuals were 0.8 and 0.64 , which were indicative of normal residuals. 
341 Table 1: The results of stochastic modeling using different Box-Jenkins steps for each precipitation 342 time series

\begin{tabular}{llcc}
\hline Station & & Tabriz & Rasht \\
\hline Normality Test & Kolmogorov-Smrinov & 0.157 & 0.000 \\
& Shapiro-Wilk & 0.399 & 0.000 \\
& & & \\
Stationarity test & ADF & -1.87 & -0.34 \\
& & & \\
Identification of & SBC & ARIMA $(0,1,2)$ & SARIMA $(0,1,1) \times(0,1,1)_{12}$ \\
model & BIC & 347.31 & 456.88 \\
& Parameters & 8.47 & 2.55 \\
Diagnostics & P-value & $\mathrm{e}_{1}=0.64, \mathrm{e}_{2}=0.5$ & $\mathrm{e}_{1}=0.96, \mathrm{E}_{12}=0.9$ \\
& P-value & $0.002,0.017$ & $0.0001,0.0001$ \\
& & 0.497 & 0.6 \\
\hline
\end{tabular}

343

344

345

346

347

348

349

350

351

352

353

\subsection{Modeling nonlinear component of precipitation time series}

In this study, different models (e.g., SVR, GEP, and GMDH) and two residual configurations were used to model the nonlinear component of the time series. A proper parameter estimation has an important role in the accuracy of precipitation forecasts. Therefore, a sensitivity analysis was conducted to identify the optimal parameters and kernel function types (e.g., Figure 3). The parameters of SVR, GEP and GMDH were summarized in Table 2, which were found from the sensitivity analysis. Some of GEP model parameters include addition (+), mutation rate (0.06), one-point recombination rate $(0.2)$, two-point recombination rate $(0.3)$, gene recombination rate (0.2) and IS transposition rate (0.2). 


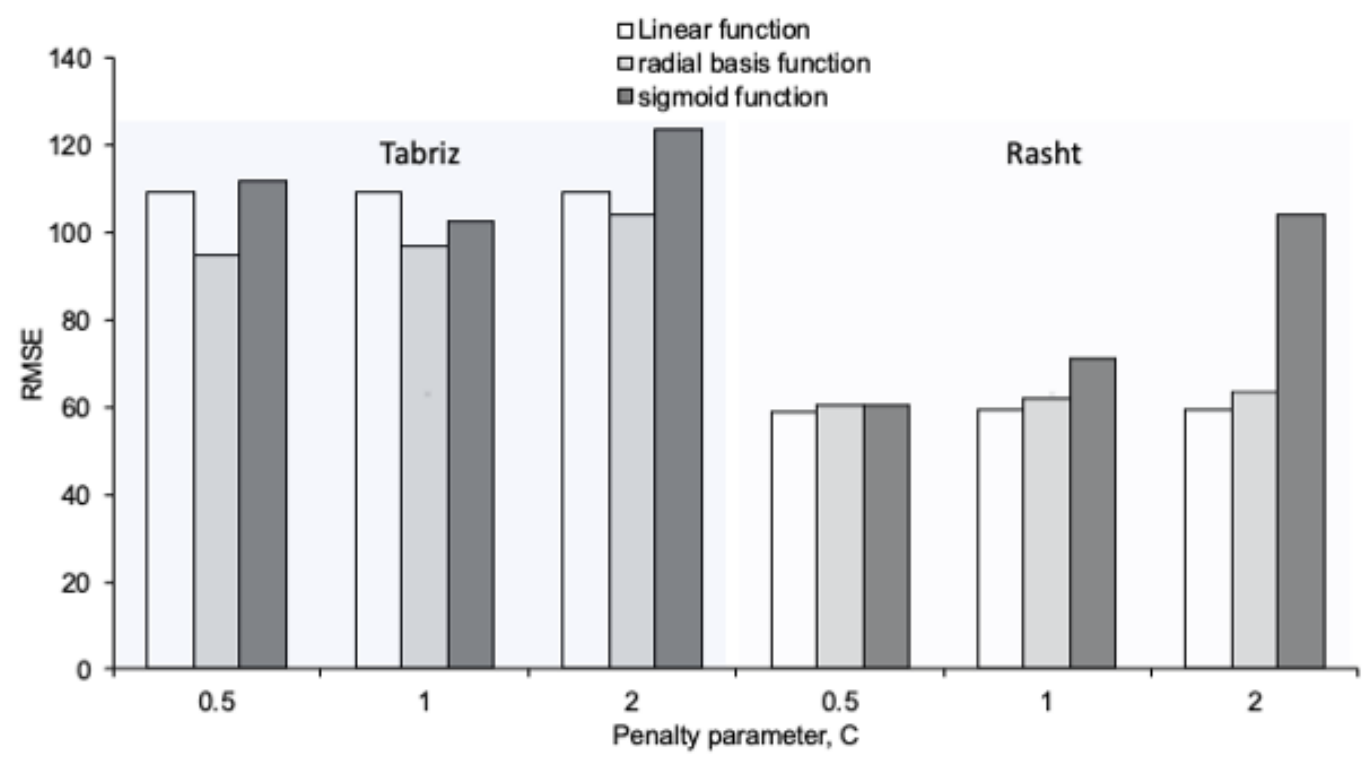

355 Figure 3: Sensitivity analysis of support vector regression model for two time series related to 356 kernel function and penalty parameter

358 Table 2: Estimated parameters for each of the individual models of Gene Expression Programming 359 (GEP), Support Vector Regression (SVR) and Group Method of Data Handling (GMDH)

\begin{tabular}{|c|c|c|c|c|c|}
\hline \multirow{3}{*}{ model } & \multirow{3}{*}{ Parameters } & \multicolumn{2}{|c|}{ Tabriz } & \multicolumn{2}{|c|}{ Rasht } \\
\hline & & \multicolumn{4}{|c|}{ Configuration } \\
\hline & & First & Second & First & Second \\
\hline \multirow{4}{*}{ GEP } & $\begin{array}{l}\text { Number of } \\
\text { generations }\end{array}$ & 1000 & 100 & 100 & 80 \\
\hline & $\begin{array}{c}\text { Chromosome } \\
\text { length }\end{array}$ & 50 & 50 & 50 & 50 \\
\hline & Head size & 8 & 7 & 7 & 7 \\
\hline & Number of genes & 4 & 3 & 3 & 3 \\
\hline \multirow[t]{2}{*}{ SVR } & Kernel function & $\begin{array}{l}\text { radial basis } \\
\text { function }\end{array}$ & sigmoid function & linear function & $\begin{array}{l}\text { radial basis } \\
\text { function }\end{array}$ \\
\hline & Penalty parameter & 0.5 & 1 & 0.5 & 2 \\
\hline \multirow{3}{*}{ GMDH } & $\begin{array}{c}\text { Maximum } \\
\text { number of } \\
\text { neurons in a layer }\end{array}$ & 5 & 10 & 10 & 10 \\
\hline & $\begin{array}{c}\text { Maximum } \\
\text { number of layers }\end{array}$ & 5 & 7 & 5 & 5 \\
\hline & Selection pressure & 0.6 & 0.5 & 0.6 & 0.1 \\
\hline
\end{tabular}

RMSE decreased by $15 \%$ when we switched the kernel function from sigmoid to radial basis

363 function with $C=0.5$ and by $8.8 \%$ when we changed the $\mathrm{C}$ parameter of the radial basis function 364 from 2 to 0.5 in the Tabriz station and for the configuration one. Another comparison was 
conducted to show the performance of single individual models with two residual configurations (Figure 4).

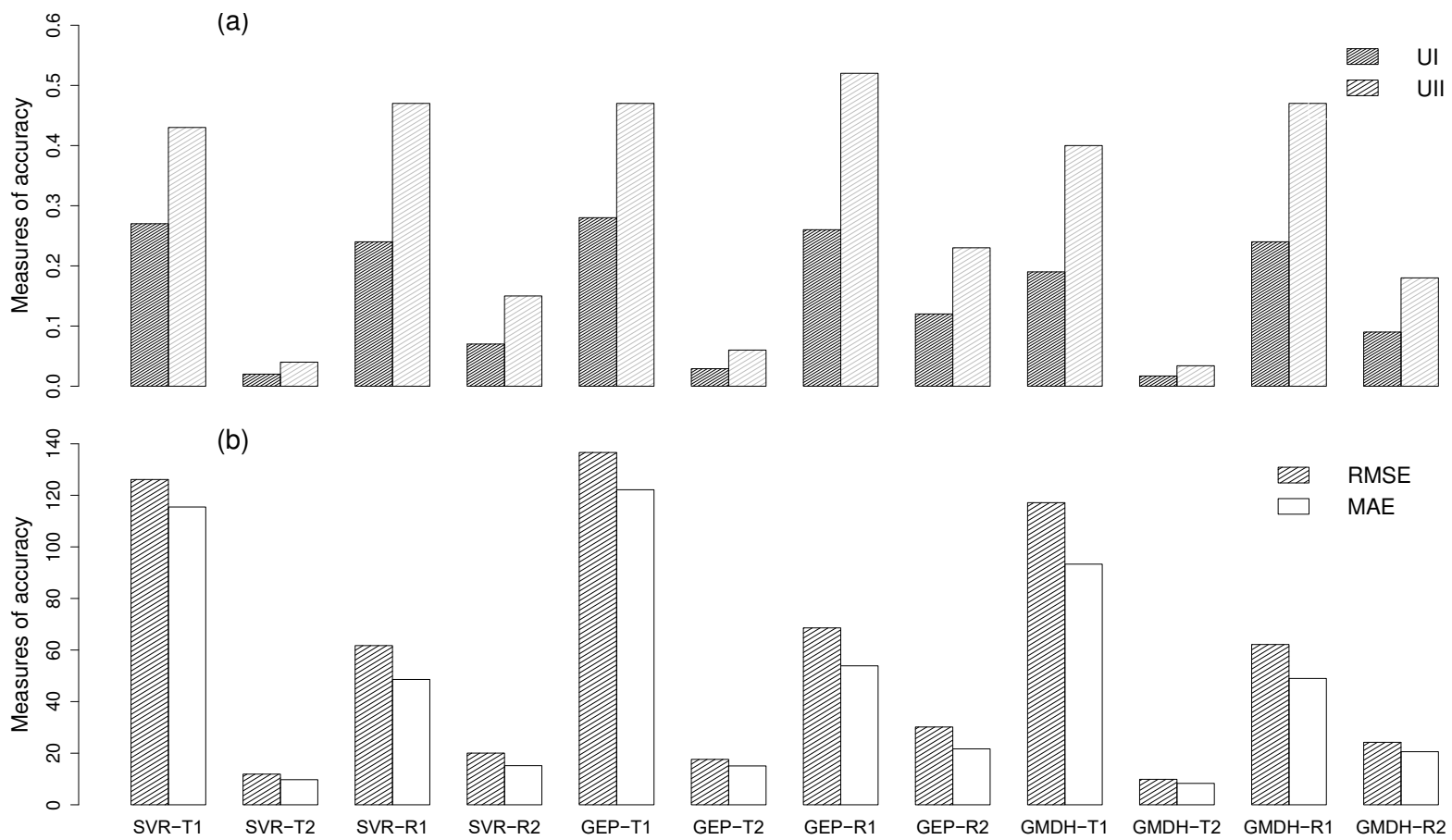

Figure 4: Comparison of the performance of two configurations for the nonlinear component of time series with single individual models and error criteria of a) Theil's coefficient of UI and UII and b) RMSE and MAE. T1, T2, R1 and R2 denote Tabriz and Rasht station for the two configurations (Table 2).

As shown in Figure 4, the error criteria decreased from configuration one to two. For instance, Theil's coefficient of UII decreased by $56 \%$ for GEP and 62\% for GMDH from configuration one to two in Rasht. By considering all models and all error criteria the average percent decreasing in Tabriz was greater than Rasht (46\%). The error criteria increased by using GEP model. For instance, MAE decreased by 5\% when GEP was replaced by SVR and by $24 \%$ when GEP was replaced by GMDH in configuration 1 in Tabriz. In Rasht, UI decreased by $42 \%$ when GEP was replaced by SVR and by $25 \%$ when GEP was replaced by GMDH in configuration two. In general, the performance of GMDH and SVR for Tabriz and Rasht precipitation forecasting was reasonably high, and the difference between the error values of SVR and GMDH for Rasht time series in configuration one is low. 
3.3. Improved hybrid models

388 The main advantage of the improved hybrid models to traditional hybrid models is to combine

389 forecasts from multiple models instead of using one single model in nonlinear part of time series.

390 The performance of improved hybrid models with different combinations and weighting methods

391 was compared against the hybrid model, composed of one linear and only one nonlinear model

392 (Table 3 and Figure 5).

393

394 Table 3: Comparison of the hybrid and improved hybrid models. Three Gene Expression 395 Programming (GEP), Support Vector Regression (SVR) and Group Method of Data Handling 396 (GMDH) were combined using four error based (Iv+SSE, MSEI+SSE, Iv+SMAPE, 397 MSEI+SMAPE) and four machine learning based methods (SVR, GA, LSR and ANN) for 398 configuration two.

\begin{tabular}{|c|c|c|c|c|c|c|c|c|c|c|c|}
\hline Model type & $\begin{array}{c}\text { Precipitation } \\
\text { time series }\end{array}$ & $\begin{array}{c}\text { Structure of } \\
\text { hybrid model }\end{array}$ & RMSE & RRMSE & MSE & RPD & APB & dm & UII & AMPE & NSE \\
\hline \multirow{3}{*}{ Hybrid } & \multirow{3}{*}{ Tabriz } & ARIMA+SVR & 11.88 & 0.041 & 141.21 & 4.5 & 0.034 & 0.86 & 0.04 & 40.83 & 0.94 \\
\hline & & ARIMA+GEP & 17.59 & 0.061 & 309.6 & 3.05 & 0.052 & 0.77 & 0.06 & 40.85 & 0.87 \\
\hline & & ARIMA+GMDH & 9.87 & 0.034 & 97.49 & 5.45 & 0.028 & $\underline{0.89}$ & 0.034 & 40.83 & 0.95 \\
\hline \multirow{8}{*}{$\begin{array}{l}\text { Improved } \\
\text { hybrid: } \\
\text { Combination } \\
\text { of nonlinear } \\
\text { models }\end{array}$} & \multirow{8}{*}{ Tabriz } & $\mathrm{Iv}+\mathrm{SSE}$ & $\overline{8.81}$ & $\overline{0.03}$ & $\overline{77.67}$ & $\overline{6.1}$ & $\overline{0.023}$ & $\overline{0.9}$ & $\overline{0.03}$ & $\overline{40.82}$ & $\overline{0.96}$ \\
\hline & & MSEI+SSE & 7.36 & 0.025 & 54.21 & 7.3 & 0.02 & 0.91 & 0.025 & 40.82 & 0.97 \\
\hline & & Iv+SMAPE & 7.73 & 0.027 & 59.9 & 6.95 & 0.023 & 0.9 & 0.026 & 40.82 & 0.97 \\
\hline & & MSEI+ SMAPE & 8.91 & 0.031 & 79.46 & 6.03 & 0.024 & 0.89 & 0.03 & 40.82 & 0.96 \\
\hline & & SVR & $\underline{5.57}$ & $\underline{0.019}$ & $\underline{31.03}$ & 9.6 & 0.017 & $\underline{0.93}$ & $\underline{0.019}$ & $\underline{40.81}$ & $\underline{0.98}$ \\
\hline & & GA & $\overline{9.39}$ & $\overline{0.032}$ & $\overline{88.31}$ & $\overline{5.7}$ & 0.025 & 0.89 & 0.032 & 40.82 & 0.96 \\
\hline & & LSR & 9.01 & 0.031 & 81.2 & 5.97 & 0.025 & 0.89 & 0.031 & 40.82 & 0.96 \\
\hline & & ANN & 7.64 & 0.026 & 58.43 & 7.04 & 0.02 & 0.91 & 0.026 & 40.82 & 0.97 \\
\hline \multirow{3}{*}{ Hybrid } & \multirow{3}{*}{ Rasht } & SARIMA+SVR & $\underline{20.02}$ & $\underline{0.187}$ & $\underline{400.9}$ & $\underline{3.77}$ & $\underline{0.142}$ & $\underline{0.97}$ & $\underline{0.15}$ & $\underline{4.58}$ & $\underline{0.92}$ \\
\hline & & SARIMA+GEP & 30.51 & 0.28 & 931.3 & 2.47 & 0.19 & 0.94 & 0.23 & 4.64 & 0.82 \\
\hline & & SARIMA+GMDH & 24.22 & 0.22 & 586.7 & 3.11 & 0.18 & 0.96 & 0.18 & 4.63 & 0.89 \\
\hline \multirow{8}{*}{$\begin{array}{l}\text { Improved } \\
\text { hybrid: } \\
\text { Combination } \\
\text { of nonlinear } \\
\text { models }\end{array}$} & \multirow{8}{*}{ Rasht } & Iv+SSE & 15.92 & 0.149 & 253.4 & 4.74 & 0.11 & 0.98 & 0.12 & 4.56 & 0.95 \\
\hline & & MSEI+SSE & 17.2 & 0.16 & 296.17 & 4.39 & 0.12 & 0.98 & 0.13 & 4.56 & 0.94 \\
\hline & & Iv+SMAPE & 16.52 & 0.154 & 272.9 & 4.57 & 0.11 & 0.98 & 0.12 & 4.55 & 0.95 \\
\hline & & MSEI+ SMAPE & 18.08 & 0.169 & 326.9 & 4.17 & 0.13 & 0.98 & 0.13 & 4.57 & 0.94 \\
\hline & & SVR & 13.03 & 0.12 & 177.56 & 5.66 & 0.091 & 0.99 & 0.1 & 4.53 & 0.96 \\
\hline & & GA & 11.48 & 0.1 & 131.84 & 6.57 & 0.078 & 0.99 & 0.088 & 4.52 & 0.97 \\
\hline & & LSR & 14.37 & 0.13 & 206.55 & 5.26 & 0.11 & $\overline{0.98}$ & 0.11 & 4.55 & 0.96 \\
\hline & & ANN & 17.7 & 0.16 & 294.99 & 4.39 & 0.1 & 0.98 & 0.13 & 4.54 & 0.94 \\
\hline
\end{tabular}



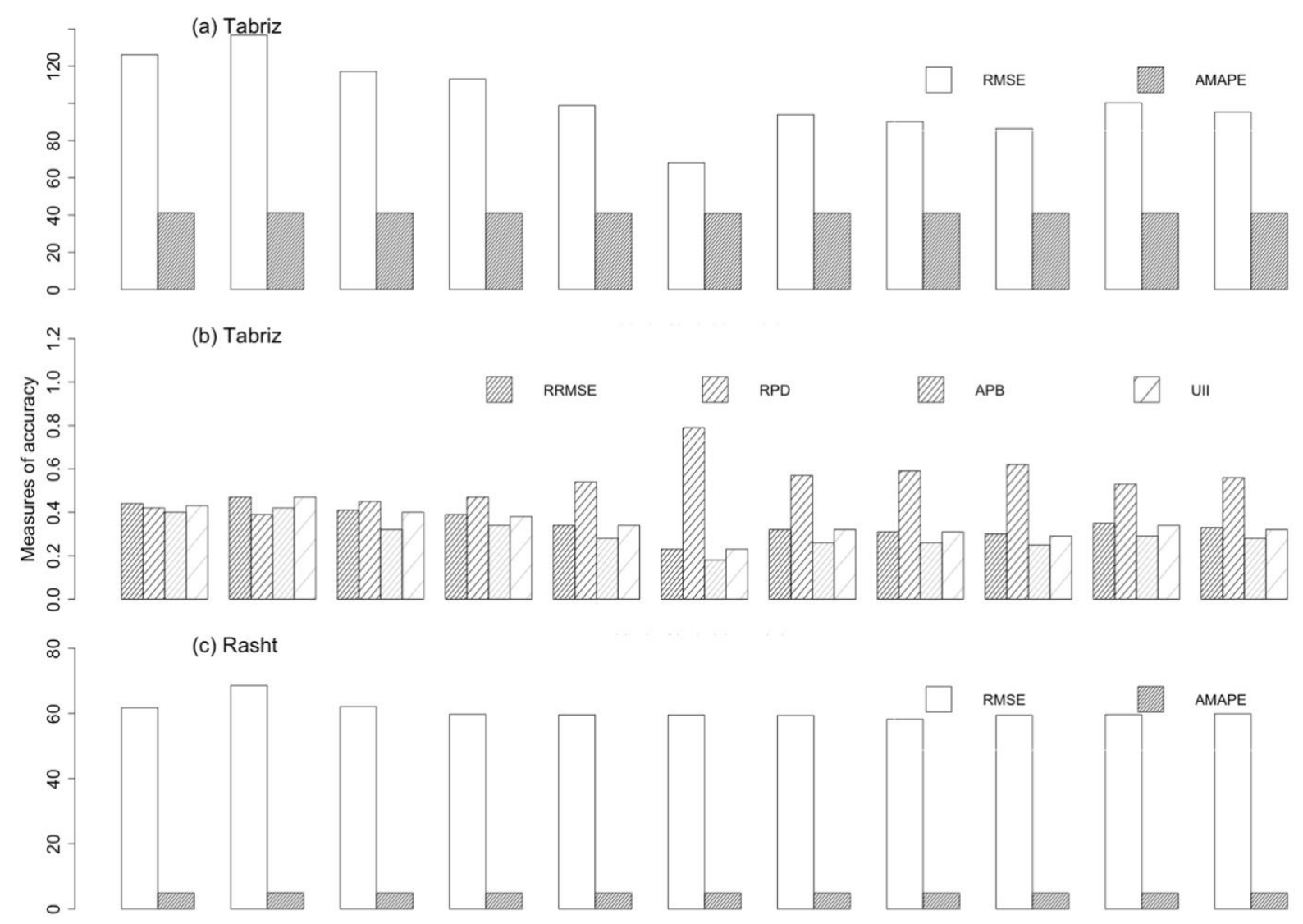

ํำ $\quad$ (d) Rasht

Figure 5: Performance of the hybrid models for configuration one and the Rasht and Tabriz stations

403 As illustrated in Figure 5, the error criteria degraded for the hybrid model using GEP as a single model (ARIMA+GEP in Table 3), relative to other single nonlinear hybrid models or combined 405 nonlinear hybrid models. The forecast accuracy was improved when the nonlinear models were 406 combined in the form of the hybrid model. For instance, in configuration two for Tabriz station, 407 RMSE improved by 5 to $43 \%$ when the ARIMA+GMDH hybrid model was replaced by the 408 proposed multi-nonlinear hybrid models such as Iv+SSE, MSEI+SSE, Iv+SMAPE, MSEI+ 409 SMAPE, SVR, GA, LSR and ANN (Table 3). The three improved hybrid models with the highest 410 performance in different stations and configurations are those combined with:1) Iv+SMAPE, GA 
411 and SVR methods for Tabriz station and configuration one; 2) SVR, MSEI+SSE and ANN methods for Tabriz station and configuration two; 3) SVR, MSEI+SMAPE and GA for Rasht station and configuration one; and 4) GA, SVR and LSR methods for Rasht station and configuration two.

In general, the hybrid models combined with SVR, GA, and the error-based methods performed better than the other methods. The average RPD criteria were 0.42 and 4.33 in the traditional hybrid model combined with one linear and one single model for Tabriz series in configurations one and two. These values were 1.17 and 3.11 for the Rasht station. The average of RPD criteria in proposed hybrid models combined with one linear and multiple nonlinear methods were improved and reached 0.58 and 6.83 for Tabriz station and 1.26 and 4.9 for Rasht station in configuration one (Figure 5) and two (Table 3). The RPD values of Rasht and Tabriz precipitation time series in configuration two are in the high range in terms of model performance (Table 3).

For evaluating the performance of hybrid models combined with multiple nonlinear methods, an AI criterion (Eq. 30) was calculated for the three best models. This calculation was compared with the best model of the traditional hybrid model. AI of Tabriz precipitation in configuration one for the hybrid models combined with Iv+SMAPE, GA and SVR methods was 44\%, 20\% and 19.3\%, respectively. AI in configuration two for the hybrid models combined with SVR, MSEI+SSE and ANN methods was 41\%, 29\% and 27\%, respectively. AI of Rasht time series in configuration one for the hybrid model combined with SVR, MSEI+SMAPE and GA methods was 6.7\%, 2.9\% and $2.7 \%$ respectively. In configuration two, AI was $44 \%, 35 \%$ and $19 \%$, respectively for the hybrid models combined with GA, SVR and LSR methods. Like other performance criteria, AI values for hybrid models combined with different methods were improved compared with other traditional hybrid models. Among the error-based combinations of three nonlinear methods, Iv+SMAPE and MSEI+SMAPE performed the best for Tabriz and Rasht stations for configuration one and MSEI+SSE and Iv+SSE for configuration two. The observed and forecasted precipitation series were shown in Figure 6 for the verification period and traditional and proposed hybrid models. 


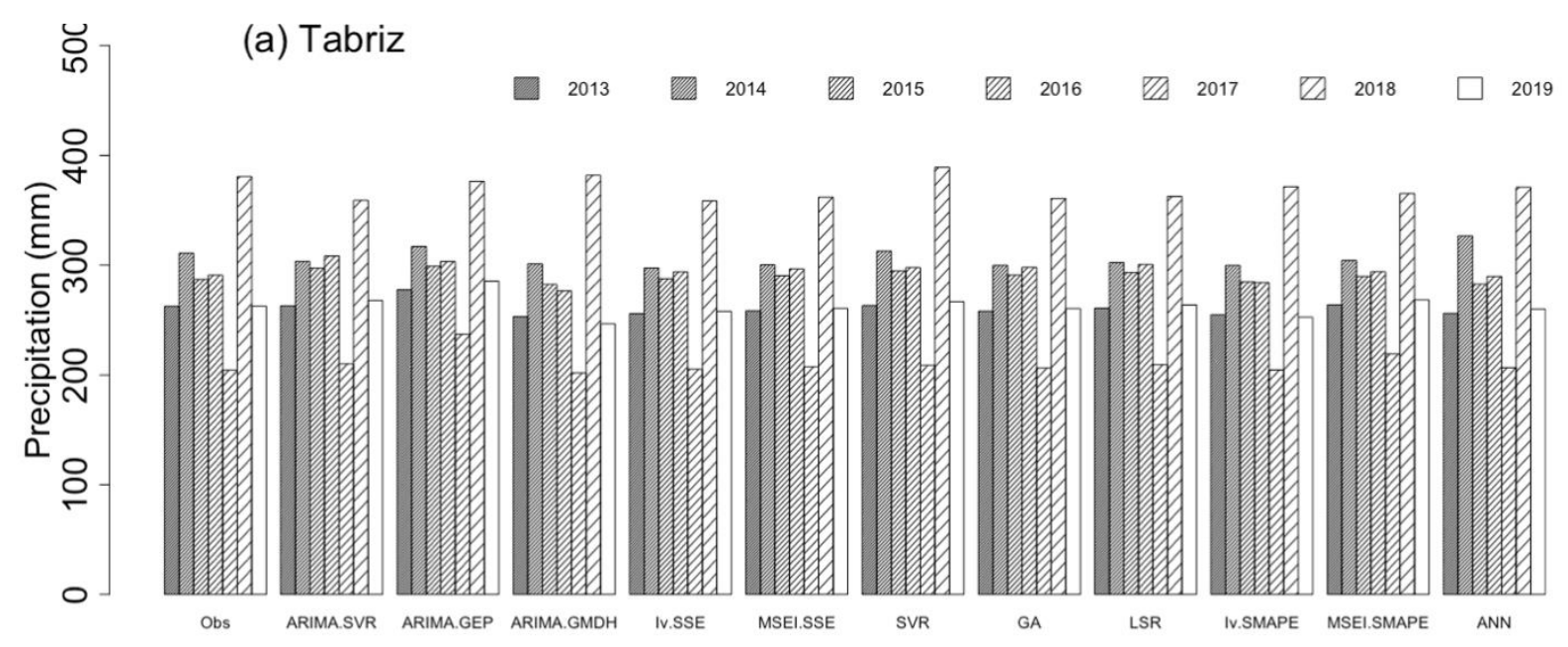

(b) Rasht

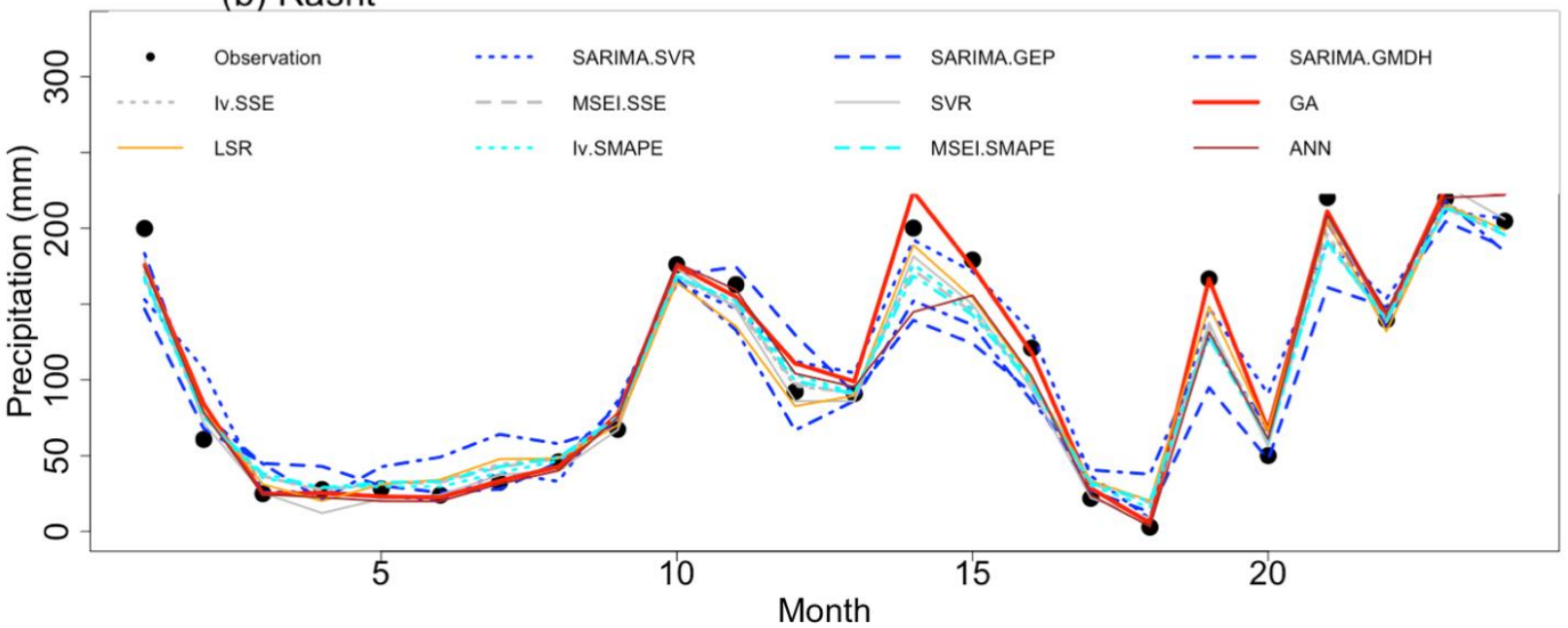

443

444

445

446

447

448

449

450

451

452

453

454

455

Figure 6: Comparison of observed and forecasted precipitation by 11 hybrid models for configuration 2for the(a) Tabriz and (b) Rasht weather stations in Iran in the verification period

The maximum and minimum annual precipitation were observed in 2018 and 2017 in Tabriz (Figure 6a), which were preserved with all improved hybrid models combined with multiple error based and other combination methods. The observed monthly average of precipitation was 107 $\mathrm{mm}$ in Rasht, which was close to the monthly average forecasted by the hybrid model combined with GA $(110 \mathrm{~mm})$. The mean annual precipitation observed was $1279 \mathrm{~mm}$ in Rasht and its associated forecasted value was $1317 \mathrm{~mm}$ according to the hybrid model combined with GA (Figure 6b). The minimum precipitation was observed in June 2019 in Rasht, and again it was preserved in all hybrid models, except for the one combined with the LSR method. The scatter plots of the two best improved hybrid models with the highest performance were illustrated in 
58

59

Figure 7 and compared against observations and the conventional hybrid methods of ARIMA+GMDH and SARIMA+SVR.

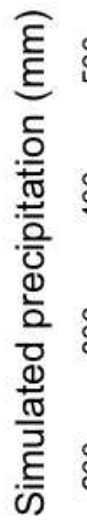

(a) Tabriz

(a) Tabriz

$\rightarrow$ ARIMA+GMDH

$\triangle$ SVR

\& - - MSEI+SSE
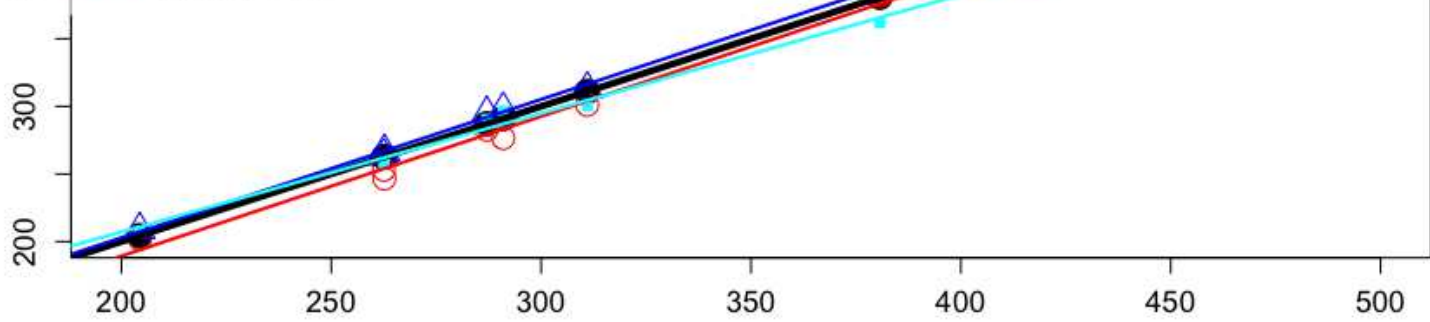

(b) Rasht

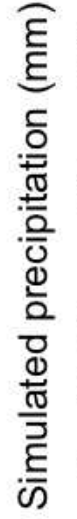

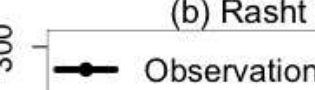

- - SARIMA+SVR

$\triangle$ SVR

¿ - $=\mathrm{GA}$

460

461

462

463

464

465

466

467

468

469

470

The R-square of the lines fitted to ARIMA+GMDH as a hybrid model and SVR and MSEI+SSE as improved hybrid model outputs were respectively 0.96, 0.99 and 0.96 in Tabriz and the R-square of the lines fitted to SARIMA+SVR as a hybrid model, and SVR and GA as improved hybrid models were respectively $0.87,0.99$ and 0.95 in Rasht. The R-square of the fitted line was increased using improved hybrid models 3 to $14 \%$. 
(a) Rasht

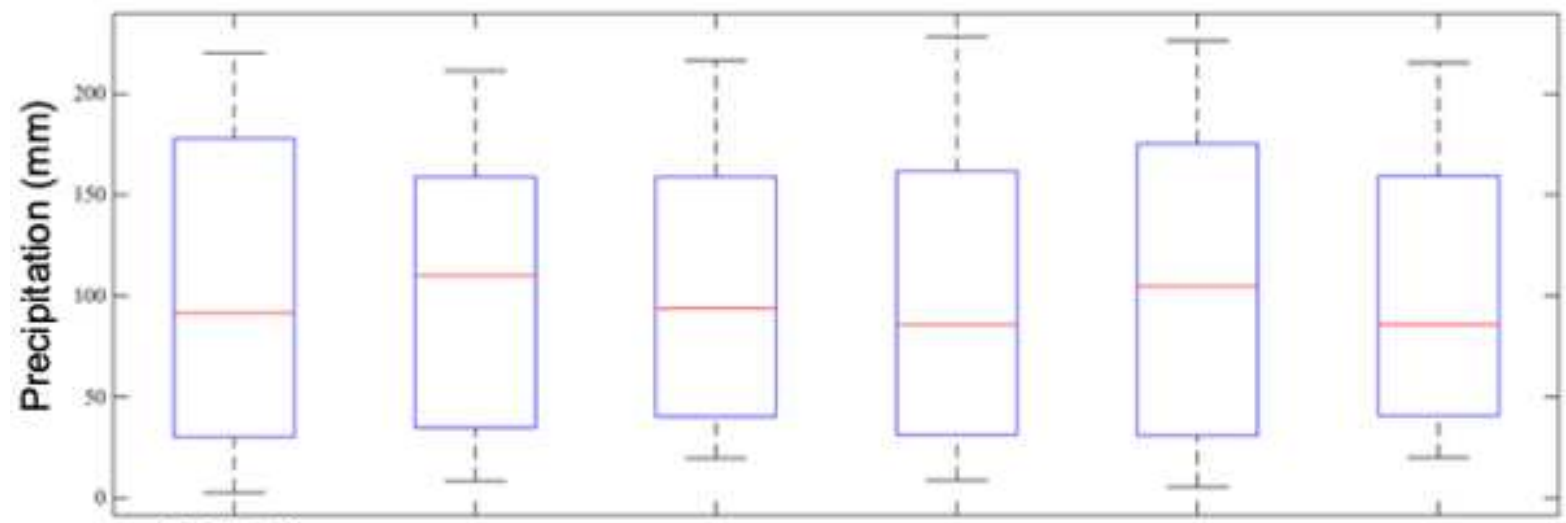

(b) Tabriz

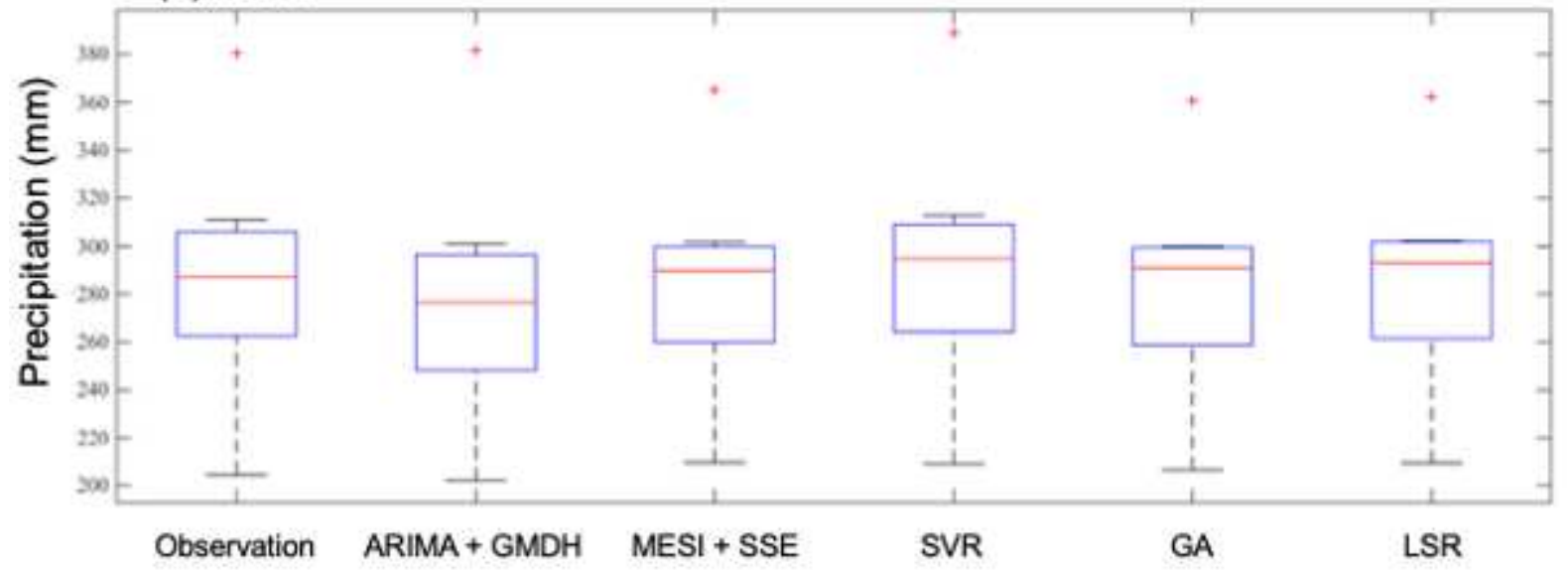

Hybrid models

472 Figure 8: Boxplot of observed and forecasted precipitation in the (a) Tabriz and (b) Rasht weather 473 stations with conventional hybrid model (ARIMA+GMDH) and four weighted hybrid models 474 combined using an error based MESI+SSE method, support vector regression (SVR), genetic 475 algorithm (GA) and least square regression (LSR)

477 The forecasts of the hybrid (e.g., ARIMA+GMDH) and improved hybrid models (e.g., 478 MESI+SSE, SVR, GA, and LSR) were compared in Figure 8. These plots showed that observed 479 precipitation matches with forecasts by the improved hybrid models (Figure 8). In Tabriz, the 480 variability of precipitation values forecasted by the hybrid model combined with SVR was similar 481 to the spread of observations. From the point of lower and upper whisker view, the high similarity was observed between observed and the hybrid model combined with SVR. The median box-plot 
of observed and proposed hybrid models had good match. The same were true for observations and forecasts by the hybrid model combined with GA for the Rasht time series (Figure 8).

\section{Discussion}

The purpose of this study was to improve the accuracy of the traditional hybrid model with one linear and one nonlinear in their structures. To achieve this, we 1) properly modeled the linear component of time series using ARIMA or SARIMA; 2) precisely selected the structures of the two configurations with antecedent residual subseries; and with antecedent observations and residual subseries and linear model simulations; 3 ) properly selected a model to represent residual subseries using an artificial intelligence technique (Liang, 2009; Zheng, 2003; Chen, et al., 2010; Moeeni et al., 2017); and 4) accurately estimated parameters of the selected model. The average RMSE, MAE, UI, and UII decreased from configuration one to two in GEP model for Tabriz and Rasht time series by $88 \%$ and 56\%, respectively; In this study, the performance of SVR and GMDH was better than GEP model. The average RMSE, MAE, UI and UII decreased by 69\%, 56\% and $61 \%$ when we switched from configuration one to two in SVR, GEP, and GMDH models for Rasht. RMSE of SVR, GEP, and GMDH models forecasts was 12, 18 and $10 \mathrm{~mm}$, respectively in configuration two for Tabriz. For estimation of monthly reference evapotranspiration in Iran, SVR had good performance than GEP (Ahmadi et al., 2021). A study investigates the performance of the multivariate regression spline, least-square support vector regression, GEP and ANN for estimation of monthly long-term rainfall and the best model was least square support vector regression (Mirabbasi et al., 2019). SVM model improved the forecasted error compared to ANN in the internal process of time variation analysis related to precipitation forecasting (Parviz, 2020). The RMSE of the GEP model forecasts decreased by $44 \%$ and $4 \%$ for configuration one when we decreased generation number from 1000 to 100 was and head size from 8 to 7 .

Hybrid models increased forecasting efficiency by combining two types of models and capturing linear and nonlinear patterns of time series. For example, in configuration one, the RMSE, RRMSE and MSE decreased by 4\%, 5\%, and 8.5\% when we replaced SARIMA with the improved hybrid model combined with SVR, by $30 \%, 25 \%$, and $44 \%$ when we replaced ARIMA with the improved hybrid model combined with GMDH. Improvement of precipitation forecasting with the hybrid model based on temporal variations, relative to SARMIA model was observed in Rasht and Gorgan 
stations in Iran with 48\% and 24\% improvements in RMSE scores (Parviz and Rasouli 2019). Furthermore, the hybrid model structure was more important in forecasting. For instance, two hybrid models based on temporal variations and decomposition of precipitation time series, which were compared with a hybrid model based on time series decomposition (linear and nonlinear components) had the minimum error (Parviz 2020).

The hybrid model performance in modeling complex phenomena has drawbacks as the nonlinearity of the decomposed time series may be high, and one model is insufficient to capture all variabilities (Mo et al., 2018). Furthermore, the error series may have high volatility and irregularity (Chen et al., 2020). Therefore, the improvement of hybrid models is necessary. The main direction of the improved hybrid model was to combine the forecast of single models with different methods instead of using one model in the nonlinear part of time series modeling. The combination methods for the integration of forecasts outperform individual models (Wang et al., 2020). All used combination methods improved the efficiency of the hybrid model. NSE improved by 0.18 with the improved hybrid model with GA in Rasht and 0.13 with the improved hybrid model with SVR in Tabriz relative to the hybrid model with GEP. Adhikari and Agrawal (2014) indicated that the combination procedure significantly outperformed all individual methods based on the evaluation scores of the weighting schemes. But it is hard to select one combination method as an inclusive method. The integration of SARIMA for the linear component of time series with a combination of SVR, GP and BP for nonlinear components improved the results of the forecasts (Mo et al., 2018). The forecasts combination aims to integrate the competing forecasts to produce an ensemble of forecasts, which is superior to each of the composing individual models (Wang et al., 2020). By combining all of the forecasts with a proper function, forecast combinations can fully use the information from each forecast model to improve the prediction accuracy and stability (Wang et al., 2019). The accuracy of combined forecasts depends on the weight of each forecast model. According to the evaluation metrics, the improved hybrid models with SVR, GA and errorbased models had high performance. In the error-based methods, the type of error equation and the inversion method were different for each configuration. Therefore, there is a need to define a comprehensive error equation and an inversion method. 
542 The average geometric standard deviation of the error for all improved hybrid models for 543 configurations (one and two) for the Tabriz and Rasht time series was (1.47-1.36) and (2.12-1.41),

544

545

546

547

548

549

550

551

552

553

554

555

556

557

558

559

560

561

562

563

564

565

566

567

568

569

570

571 respectively, which indicates forecasts overestimation. If the median of annual Tabriz time series were calculated in the verification period for all improved hybrid models, the median of all time series (observed, improved hybrid model) occurred in 2015. Based on the improved hybrid model using GA, the months with the high and low errors were calculated. The high error was related to May $($ RRMSE $=0.167)$, and low was related to July $($ RRMSE $=0.0044)$. Based on the forecasted monthly time series, the seasonal time series was calculated. The maximum observed precipitation occurred in autumn, and it was preserved in improved hybrid models.

\section{Conclusion}

Analysis of precipitation time series can provide insight into its spatial and temporal complexity. This study introduced a few hybrid models, which first separate linear and nonlinear components of precipitation and then extract the temporal patterns with high-performing machine learning methods for the nonlinear component of the precipitation in two weather stations in humid and semiarid climates of Iran. The hybrid model is a combination of stochastic approaches and one of the machine learning techniques. The type of artificial intelligence techniques can affect the forecasts of the hybrid model. We found that the improved hybrid models combined by SVR and GMDH showed better performance than GEP in Rasht and Tabriz weather stations.

In contrast to the traditional hybrid models used for precipitation forecast, a combination of forecasts by multiple nonlinear models was used in the structure of hybrid models used in this study instead of using only one nonlinear model. An ensemble of forecasts using an appropriate weighting method and using artificial intelligence techniques improved forecast errors for annual and monthly precipitation. This was shown by all of the forecast combination methods. Among combination methods, artificial intelligence techniques (SVR), optimization method (GA), and error based methods showed the highest performance. In the error-based methods, the type of error formulation and the structure of the inversion method had the most crucial role. In general, three factors have the main control on the precipitation ensemble forecast using data-driven methods used in this study: separating linear and nonlinear components and appropriately modeling the nonlinear component; Assigning appropriate weights to each ensemble member of the nonlinear 
572 modeling; and finally, the type of nonlinear models. One of the advantages of the improved hybrid

573 model is that it can extract information with high confidence. It uses an accurate forecast

574 combination method embedded in its structure that can overcome the challenges in modeling

575 nonlinear processes in real time series. The other advantage is that it uses optimization methods

576 to estimate ANN and SVR parameters and assign weights to the ensemble members. The proposed

577 improved hybrid model can be a powerful tool to increase precipitation forecast confidence across

578 time scales by combining forecasts from sophisticated machine learning methods instead of relying

579 on only individual models.

$580 \quad$ Funding Statement

581 This research received no external funding.

582

583

Author's Contribution

584

585

586

587

588

589

590

591

592

593

594

595

596

597

598

599

L.P. and K.R. analyzed the data and wrote the paper. A.T.H., L.P. and K.R. Research conceptualization and methodology, supervision, reviewing and editing A.T.H.

\section{Availability of data and material}

The data that support the findings of this study is available from the corresponding author upon request.

\section{Code availability}

Not applicable.

\section{Declarations}

Ethics approval The authors confirm that this article is original research and has not been published or presented previously in any journal. This study does not include any animal experiments.

Consent to participate Not applicable.

Consent for publication The authors agree with the publisher to publish this work.

600

601 References

602 Abdelbaki AM (2016) Using automatic calibration method for optimizing the performance of

603 pedotransfer functions of saturated hydrolic conductively. Ain Shams Eng J 7:653-662.

604 https://doi.org/10.1016/j.asej.2015.05.012

605 Adhikari R, Agrawal RK (2014) Performance evaluation of weights selection schemes for linear

606 combination of multiple forecasts. Artif Intell Rev 42(4):529-548. https://doi.org/10.1007/s10462-

$607 \quad \underline{012-9361-Z}$ 
Ahmadi F, Mehdizadeh S, Mohammadi B, Pham QB, Doan TNC, Vo ND (2021) Application of an artificial intelligence technique enhanced with intelligent water drops for monthly reference evapotranspiration estimation. Agric Water Manag 244:106622. https://doi.org/10.1016/j.agwat.2020.106622

Bouznad IE, Guastaldi E, Zirulia A, Brancale M, Barbagli A, Bengusmia D (2020) Trend analysis and spatiotemporal prediction of precipitation, temperature, and evapotranspiration values using the ARIMA models: case of the Algerian Highlands. Arab J Geosci 13(24):1-17. https://doi.org/10.1007/s12517-020-06330-6

Box GEP, Jenkins GM (1976) Times series analysis-forecasting and control. Englewood Cliffs, NJ: Prentice-Hall.

Chen KY, Wang CH (2007) A hybrid SARIMA and support vector machines in forecasting the production values of the machinery industry in Taiwan. Expert Syst Appl 32(1):254-264. https://doi.org/10.1016/j.eswa.2005.11.027

Chen S, Pao-Shan Y, Yi-Hsuan T (2010) Statistical downscaling of daily precipitation using support vector machines and multivariate analysis. J Hydrol 385(1-4):13-22. https://doi.org/10.1016/j.jhydrol.2010.01.021

Chen X, Zhu S (2013) Improved hybrid model based on support vector regression machine for monthly precipitation forecasting. J Comput 8(1), 232-238. https://doi.org/10.4304/jcp.8.1.232-239

Chen W, Xu H, Chen Z, Jiang M (2020) A novel method for time series prediction based on error decomposition and nonlinear combination of forecasters. Neurocomputing 426:85-103. https://doi.org/10.1016/j.neucom.2020.10.048

Danandeh Mehr A, Nourani V, Karimi Khosrowshahi V, et al. (2019) A hybrid support vector regression-firefly model for monthly rainfall forecasting. Int J Environ Sci Technol 16:335-346. https://doi.org/10.1007/s13762-018-1674-2

De Martonne E (1925) Traité Géographie. Physique: 3 tomes. Max leclcrc and H. Bourrclier, proprietors of Librairic Armard Colin: Paris.

Deng S, Yuan C, Yang L, Zhang L (2018) Distributed electricity load forecasting model mining based on hybrid gene expression programming and cloud computing. Pattern Recognit Lett 109:72-80. https://doi.org/10.1016/j.patrec.2017.10.004

Díaz-Robles LA, Ortega JC, Fu JS, Reed GD, Chow JC, Watson JG, Moncada-Herrera JA (2008) A hybrid ARIMA and artificial neural networks model to forecast particulate matter in urban areas: 
639 The case of Temuco, Chile. Atmos Environ 42(35):8331-8340.

640 https://doi.org/10.1016/j.atmosenv.2008.07.020

641 Duveiller G, Fasbender D, Meroni M (2016) Revisiting the concept of a symmetric index of 642 agreement for continuous datasets. Sci Rep 6(1):1-14. https://doi.org/10.1038/srep19401

643 Emberger L (1952) Sur le quotient pluviothermique. Comptesrendushebdomadaires des séances 644 de l'académie des sciences 234 (26):2508-10.

645 Ferreira C (2001) Gene expression programming: a new adaptive algorithm for solving problems. 646 Complex Syst 13(2):87-129.

647 Frietas PSA, Rodrigues AJL (2006) Model combination in neural-based forecasting. Eur J Oper 648 Res 173(3):801-814. https://doi.org/10.1016/j.ejor.2005.06.057

649 Hamidi O, Poorolajal J, Sadeghifar M, Abbasi H, Maryanaji Z, Faridi HR, Tapak L (2015) A 650 comparative study of support vector machines and artificial neural networks for predicting 651 precipitation in Iran. Theor Appl Climatol 119(3):723-731. https://doi.org/10.1007/s00704-014-1141$652 \underline{z}$

653 Hurst H, Black R, Simaik Y (1965) Long-term storage: an experimental study. Constable, London.

654 Jeddi S, Sharifian S (2020) A hybrid wavelet decomposer and GMDH-ELM ensemble model for 655 Network function virtualization workload forecasting in cloud computing. Appl Soft Comput 656 88:105940. https://doi.org/10.1016/j.asoc.2019.105940

657 Marzocca P, Nichols JM, Molanese A, Seaver M, Trickey ST (2008) Second-order spectra for 658 quadratic nonlinear systems by Volterra functional series: Analytical description and numerical 659 simulation. Math Control Signals Syst 22:1882-1895. https://doi.org/10.1016/j.ymssp.2008.02.002

660 Kolmogorov AN (1933) Sulla determinazioneempirica di una legge di distribuzione, Giornale dell 661 Instituto ItalianodegliAttuaru 4:83-91.

662 Liang YH (2009) Combining seasonal time series ARIMA method and neural networks with 663 genetic algorithms for predicting the production value of the mechanical industry in Taiwan. 664 Neural Comput Appl 18(7):833-841. https://doi.org/10.1007/s00521-008-0216-0

665 Liu Y, Lian J, Bartolacci MR, Zeng QA (2014) Density-based penalty parameter optimization on C-SVM. 666 Sci World J. https://doi.org/10.1155/2014/851814

667 Mehdizadeh S, Behmanesh J, Khalili K (2017) A comparison of monthly precipitation point 668 estimates at 6 locations in Iran using integration of soft computing methods and GARCH time 669 series model. J Hydrol 554:721-742. https://doi.org/10.1016/j.jhydrol.2017.09.056 
Mirabbasi R, Kisi O, Sanikhani H, Meshram SG (2019) Monthly long-term rainfall estimation in Central India using M5Tree, MARS, LSSVR, ANN and GEP models. Neural Comput Appl 31(10):6843-6862. https://doi.org/10.1007/s00521-018-3519-9

Mo L, Xie L, Jiang X, Teng G, Xu L, Xiao J (2018) GMDH-based hybrid model for container throughput forecasting: Selective combination forecasting in nonlinear subseries. Appl Soft Comput 62:478-490. https://doi.org/10.1016/j.asoc.2017.10.033

Moeeni H, Bonakdari H, Fatemi SE, Zaji AH (2017) Assessment of stochastic models and a hybrid artificial neural network - genetic algorithm method in forecasting monthly reservoir inflow. INAE Lett 2:13-23. https://doi.org/10.1007/s41403-017-0017-9

Moravej M, Amani P, Hosseini-Moghari SM (2020) Groundwater level simulation and forecasting using interior search algorithm-least square support vector regression (ISA-LSSVR). Groundw Sustain Dev 11:100447. https://doi.org/10.1016/j.gsd.2020.100447

Murthy KV, Narasimha R, Saravana A, Vijaya Kumar K (2018) Modeling and forecasting rainfall patterns of southwest monsoons in North-East India as a SARIMA process. Meteorol and Atmos Phys 130(1):99-106. https://doi.org/10.1007/s00703-017-0504-2

Nugroho A, Hartati S, Mustofa K (2014) Vector Autoregression (Var) Model for Rainfall Forecast and Isohyet Mapping in Semarang-Central Java-Indonesia. Inter J Adv Comput Sci Appl 5(11):44-49. https://doi.org/10.14569/IJACSA.2014.051108

Nwokike CC, Offorha BC, Obubu M, Ugoala CB, Ukomah HI (2020) Comparing SANN and SARIMA for forecasting frequency of monthly rainfall in Umuahia. Sci Afr 10:e00621. https://doi.org/10.1016/j.sciaf.2020.e00621

Papalaskaris T, Panagiotidis T, Pantrakis A (2016) Stochastic monthly rainfall time series analysis, modeling and forecasting in Kavala City, Greece, North-Eastern Mediterranean Basin. Procedia Eng 162:254-263. https://doi.org/10.1016/j.proeng.2016.11.054

Parviz L, Rasouli K (2019) Development of precipitation forecasts model based on artificial intelligence and subseasonal clustering. J Hydrol Engin 24(12):04019053. https://doi.org/10.1061/(ASCE)HE.1943-5584.0001862

Parviz L (2020) Comparative evaluation of hybrid SARIMA and machine learning techniques based on time varying and decomposition of precipitation time series. J Agric Sci Technol 22(2):563-578. 
Prudêncio R, Ludermir T (2006) A machine learning approach to define weights for linear combination of forecasts. In International Conference on Artificial Neural Networks (Springer, Berlin, Heidelberg 274-283.

Rasouli K, Hsieh WW, Cannon AJ (2012) Daily streamflow forecasting by machine learning methods with weather and climate inputs. J Hydrol 414:284-293. https://doi.org/10.1016/j.jhydrol.2011.10.039

Rasouli K, Nasri BR, Soleymani A, Mahmood TH, Hori M, Haghighi AT (2020) Forecast of streamflows to the Arctic Ocean by a Bayesian neural network model with snowcover and climate inputs. Hydrol Res 51(3):541-561. https://doi.org/10.2166/nh.2020.164

Rostami A, Kamari A, Panacharoensawad E, Hashemi A (2018) New empirical correlations for determination of Minimum Miscibility Pressure (MMP) during N2-contaminated lean gas flooding. J Taiwan Inst Chem Eng 91:369-382. https://doi.org/10.1016/j.jtice.2018.05.048

Ruiz-Aguilar JJ, Turias IJ, Jiménez-Come MJ, Cerbán MM (2014) Hybrid approaches of support vector regression and SARIMA models to forecast the inspections volume. International Conference on Hybrid Artificial Intelligence Systems 502-514.

Said ES, Dickey DA (1984) Testing for unit roots in autoregressive-moving average models of unknown order. Biometrika 71(3):599-607. https://doi.org/10.1093/biomet/71.3.599

Shapiro SS, Wilk MB (1965) An analysis of variznce test for normality (complete samples). Biometrika 52(3-4):591-611. https://doi.org/10.1093/biomet/52.3-4.591

Shenify M, Danesh AS, Gocić M, Taher RS, Wahab AWA, Gani A, Shamshirband S, Petković D (2016) Precipitation estimation using support vector machine with discrete wavelet transform. Water Resour Manag 30(2):641-652. https://doi.org/10.1007/s11269-015-1182-9

Song C, Xiaoshuang F (2020) Research on different weight combination in air quality forecasting models. J Clean Prod 261:121169. https://doi.org/10.1016/j.jclepro.2020.121169

Theil H (1961) Economic forecasts and policity. North -Holland Pub. Co.

Theil H (1966) Applied economic forecasting. North- Holland Pub. Co.

Timmermann A (2006) Forecast combinations, in: G. Elliott, C. Granger, A. Timmermann (Eds.), Handbook of Economic Forecasting, Elsevier, pp 135-196.

Vapnik V (1995) The Nature of Statistical Learning Theory. Springer science \& business media. Volterra V (1959) Theory of Functionals and of Integral and Integro-Differential Equations, Dover, Inc., New York, 1959 Reprint of 1930 
731 Wang, Sh., Feng, J., Liu, G. 2013. Application of seasonal time series model in the precipitation

732 forecast. Math Comput Model 58(3-4):677-683. https://doi.org/10.1016/j.mcm.2011.10.034

733 Wang J, Wang Z, Li X, Zhou H (2019) Artificial bee colony-based combination approach to 734 forecasting agricultural commodity prices. Inter $\mathrm{J}$ Forecast.

735 https://doi.org/10.1016/j.ijforecast.2019.08.006

736 Wang J, Zhou H, Hong T, Li X, Wang S (2020) A multi-granularity heterogeneous combination 737 approach to crude oil price forecasting. Energy Econ 91:104790.

738 https://doi.org/10.1016/j.eneco.2020.104790

739 Winkler RL, Makridakis S (1983) The combination of forecasts. J R Stat Soc Ser A Stat Soc 740 146(2):150-157. https://doi.org/10.2307/2982011

741 Xu S, Chan HK, Zhang T (2019) Forecasting the demand of the aviation industry using hybrid 742 time series SARIMA-SVR approach. Transp Res E: Logist. Transp Rew 122:169-180. 743 https://doi.org/10.1016/j.tre.2018.12.005

744 Yoon H, Kim Y, Ha K, Lee SH, Kim GP (2017) Comparative evaluation of ANN-and SVM-time 745 series models for predicting freshwater-saltwater interface fluctuations. Water 9(5):323. 746 https://doi.org/10.3390/w9050323

747 Yousefi P, Shabani S, Mohammadi H, Naser G (2017) Gene expression programing in long term 748 water demand forecasts using wavelet decomposition. Procedia Eng 186:544-550. 749 https://doi.org/10.1016/j.proeng.2017.03.268

750 Zhang GP (2003) Time series forecasting using a hybrid ARIMA and neural network model. 751 Neurocomputing 50:159-175. https://doi.org/10.1016/S0925-2312(01)00702-0

752 Zhu S, Lian X, Wei L, Che J, Shen X, Yang L, Qiu X, Liu X, Gao W, Ren X, Li J (2018) PM 2.5 753 forecasting using SVR with PSOGSA algorithm based on CEEMD, GRNN and GCA considering 754 meteorological factors. Atmos Environ 183:20-32. https://doi.org/10.1016/j.atmosenv.2018.04.004 